\title{
Evaluation of Residual Stresses Introduced by Laser Shock Peening in Steel using Different Measurement Techniques
}

\author{
D. Glaser ${ }^{1,2, a *}$, M. Newby ${ }^{3, b}$, C. Polese ${ }^{2,4, c}$, L. Berthe ${ }^{5, d}$, A.M. Venter ${ }^{4,6, e}$, \\ D. Marais ${ }^{6, f}$, J.P. Nobre ${ }^{2, g}$, G. Styger ${ }^{7, h}$, S. Paddea ${ }^{8, i}$ and S.N. van Staden ${ }^{2, j}$ \\ ${ }^{1}$ CSIR National Laser Centre, Brummeria, Pretoria, South Africa \\ ${ }^{2}$ University of the Witwatersrand, Johannesburg, South Africa \\ ${ }^{3}$ Eskom Holdings SOC Ltd, Johannesburg, South Africa \\ ${ }^{4}$ DST-NRF Centre of Excellence in Strong Materials, University of the Witwatersrand, \\ Johannesburg, 2000, South Africa \\ ${ }^{5}$ Laboratoire PIMM (ENSAM, CNRS, CNAM, Hesam Université), Paris, France \\ ${ }^{6}$ South African Nuclear Energy Corporation (Necsa) SOC Limited, Pretoria, 0001, South Africa \\ ${ }^{7}$ University of Johannesburg, Auckland Park, Johannesburg, South Africa \\ ${ }^{8}$ The Open University, Milton Keynes, United Kingdom \\ aDDGlaser@csir.co.za, 'b Mark.Newby@eskom.co.za, 'Claudia.Polese@wits.ac.za, \\ 'Laurent.Berthe@ensam.eu, ${ }^{\mathrm{e} A n d r e w . V e n t e r @ n e c s a . c o . z a, ~ f D e o n . M a r a i s @ n e c s a . c o . z a, ~}$ \\ gJoaoPaulo.Nobre@wits.ac.za, 'haryStyger@gmail.com, 'S.Paddea@open.ac.uk, \\ 'Sean.vanStaden2@students.wits.ac.za
}

Keywords: Laser Shock Peening, Residual Stress, Synchrotron Diffraction, Incremental-Hole Drilling, Neutron Diffraction, Contour Stress Measurement

\begin{abstract}
The development of a residual stress engineering technology such as laser shock peening (LSP) requires evaluation of the process by quantification of the desired effect. Applications of LSP for turbine blade integrity enhancement due to expected deeper compressive residual stresses with lower surface roughness compared to conventional shot peening (SP), have resulted in the analysis of LSP on $12 \mathrm{CrNiMoV}$ steel samples. The investigation compares different residual stress measurement techniques such as energy dispersive synchrotron X-ray diffraction (SXRD), laboratory X-ray diffraction (XRD) with sequential electro-polishing, neutron diffraction (ND), incremental-hole drilling (IHD), and the contour method (CM). This study highlights the benefits and opportunities of using complimentary residual stress measurement techniques in order to gain insight into the residual stresses within a material.
\end{abstract}

\section{Introduction}

Laser shock peening (LSP) is a residual stress engineering technology specifically used to introduce beneficial compressive residual stresses into critical components to enhance fatigue and/or stress corrosion cracking (SCC) performance [1]. The current research has been conducted within a program focused toward development of LSP technology for applications of low pressure (LP) steam turbine blades which are typically susceptible to fatigue and SCC specifically in the blade attachment region as depicted in Fig. 1 [2]. Conventionally the highly stressed fir-tree attachment locations are mechanically shot peened (SP), however the laser-based LSP technology offers various benefits such as deeper levels of compressive residual stresses with improved surface roughness, as well as potentially being more suitable for reliable application to complex 3D surfaces. An integral aspect of the development of a residual stress 
engineering technology such as LSP is quantification of the residual stresses introduced. This study explores the attributes of residual stress measurements conducted using synchrotron X-ray diffraction (SXRD), laboratory X-ray diffraction (XRD) in conjunction with electro-polishing, fine incremental-hole drilling (IHD), neutron diffraction (ND) and the contour method (CM).

\section{Methodology}

Sample generation: Samples were extracted from an ex-service LP steam turbine blade by removing slices from the fir-tree attachment region as depicted in Fig. 1. A stress relieving cycle of $660^{\circ} \mathrm{C}$ for 20 minutes was performed on the coupons. The samples were wire EDM cut to dimensions of $20 \times 20 \times 15 \mathrm{~mm}^{3}$ and the surfaces ground. Electro-polishing was used to remove the surface grinding effects and hardness checked to ensure that the stress relieving did not alter the mechanical properties. Laboratory XRD measurements were performed on each sample before and after LSP processing to evaluate repeatability of the sample preparation and LSP processing. The work presented in this paper only considers the stresses in the y-direction as per the schematic in Fig. 1. Stresses are computed from the measured strains by utilizing a Young's modulus of $204 \mathrm{GPa}$ and Poisson's ratio of 0.3. In the case of the XRD analyses the X-ray elastic constants were experimentally determined. The uncertainties associated with the diffraction based measurements of SXRD, and ND was typically less than $\pm 10 \mathrm{MPa}$ and XRD less than \pm 15 $\mathrm{MPa}$, however these have been omitted from the presented results for purposes of clarity.

LSP processing: Initial proof-of-concept LSP processing was performed at the CSIR National Laser Centre in order to determine approximate LSP parameter combinations. Final processing was performed at the PIMM Laboratory (ENSAM-CNRS-CNAM) due to enhanced beam quality and an additional study that included parameter investigations, such as spot size effect, that require a high energy laser source. A Thales GAIA laser operating at a wavelength of $532 \mathrm{~nm}$ was used with sample immersion in a water tank. A black PVC tape (around $100 \mu \mathrm{m}$ thick with a $30 \mu \mathrm{m}$ adhesive) was employed as a sacrificial thermo-protective overlay, and the laser spot overlap was kept constant at $21.5 \%$. Using XRD as a screening analysis, an appropriate power intensity of $5 \mathrm{GW} / \mathrm{cm}^{2}$ with a $2 \mathrm{~mm}$ spot size was identified as optimal parameters. The LSP processing was performed on the $20 \times 20 \mathrm{~mm}^{2}$ sample face with a $10 \times 10 \mathrm{~mm}^{2}$ LSP patch as depicted in Fig. 1 (except for a sample with an $18 \times 18 \mathrm{~mm}^{2}$ LSP patch specifically for neutron diffraction measurements). Only one application layer of LSP was used on the samples.

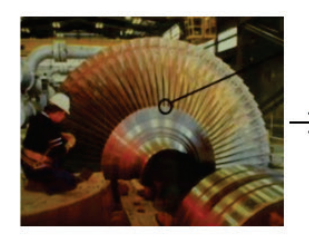

Low Pressure Steam turbine rotor

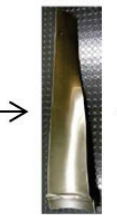

turbine blade

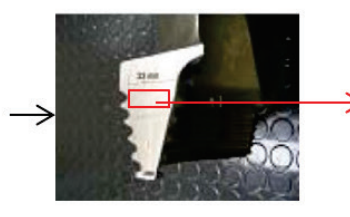

Fir tree attachment location

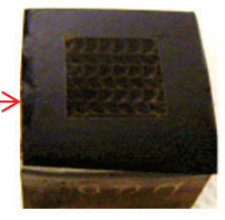

LSP Coupon with coating LSP Coupon after after processing removal of coating

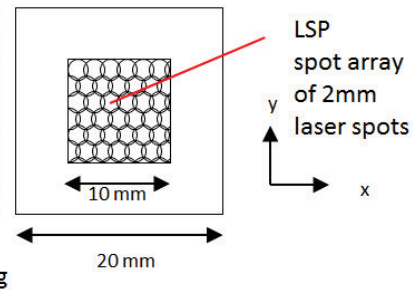

famples within the LP blade and a schematic of the LSP processed coupons.

Laboratory X-ray diffraction (XRD): The residual stress analysis was performed using conventional laboratory XRD measurements with a Proto iXRD (Proto Manufacturing Inc., Taylor, Michigan USA) instrument. A Cr-Ka X-ray source with a wavelength of $2.291 \AA$ was used in conjunction with a round $1.0 \mathrm{~mm}$ aperture. Reflections from the (211) peak for the steel were used, where the Bragg angle was located at $156.31^{\circ}$. The measurements were performed as per the $\sin ^{2} \psi$ technique, whereby 20 exposures of 1 second each were used for $7 \psi$ measurement angles per strain measurement. Measurements were performed at 0, 45, and 90 degrees in order to obtain the principal stresses. Stresses were calculated using the X-ray elastic constants 
measured using a 4-point bend apparatus as per the ASTM 146-91 designation which rendered $-\mathrm{S}_{1}=1.115 \times 10^{-6} \mathrm{MPa}^{-1}$ and $1 / 2 \mathrm{~S}_{2}=5.247 \times 10^{-6} \mathrm{MPa}^{-1}$. Sequential layer removal and XRD measurements were performed in order to obtain the through-thickness stress variation. A Struers Lectro-Pol 5 was used to perform electro-polishing to remove around 15 to 100 microns of material per step, with finer increments used nearer to the surface. A correction factor proposed by Moore and Evans incorporated in the Proto software has been applied to the measured stresses in order to correct for the material removal [3].

Synchrotron X-ray diffraction (SXRD): The SXRD measurements were conducted at the ID15A beamline (experiment ME1440) at the ESRF facility in Grenoble, France. Energydispersive measurements were performed with $300 \mathrm{keV}$ X-rays which allowed for transmission through the $20 \mathrm{~mm}$ dimension of the samples in order to provide strain measurements in the $\mathrm{y}$ direction as indicated in the Fig. 1 schematic. The beam dimensions were set to 50 microns (orientated through the thickness) by 100 microns, and a diffracting angle of around $3^{\circ}$ results in a gauge volume elongation to around $1.9 \mathrm{~mm}$ along the sample surface. A GSAS Pawley analysis was used in to determine the lattice parameter $\mathrm{d}_{0}$ accounting for multiple peaks for the BCC material. A small pillar of the material $\left(2 \times 2 \times 10 \mathrm{~mm}^{3}\right)$ was used to determine the stressfree lattice parameter.

Neutron diffraction (ND): The ND measurements were performed at the neutron strain scanner instrument MPISI located at the SAFARI-1 research reactor of Necsa (South African Nuclear Energy Corporation). Using a monochromatic wavelength of $1.67 \AA$ the $\mathrm{Fe}(211)$ reflection manifests at a diffraction angle $(2 \theta)$ of $\sim 90^{\circ}$, which in conjunction with beam limiting apertures, defined an elongated cuboid gauge volume of $0.3 \times 0.3 \times 17 \mathrm{~mm}^{3}$. To account for the $\mathrm{d}_{0}$ value, a bi-axial stress condition was assumed in which the normal stress component is zero [6]. From this approach the $\mathrm{d}_{0}$ value was determined at each measurement position and used for point-by-point strain calculation. Measurements were taken at intervals of $0.15 \mathrm{~mm}$, starting at $0.25 \mathrm{~mm}$ from the peened surface to avoid partial filling of the gauge volume.

Incremental-hole drilling (IHD): The IHD has been performed using a SINT MTS3000 instrument which uses a high speed inverted cone carbide end mill. Type A strain gauges (CEA13-062UL-120) have been used which have a nominal hole diameter of $2 \mathrm{~mm}$, and stresses are reported to $50 \%$ of the measured hole diameter of $1.75 \mathrm{~mm}$. The drilling was performed with 60 increments of $0.02 \mathrm{~mm}$ each, and the stresses calculated as per the ASTM E837-13 EXT formulation for non-uniform residual stresses. The data was computed using the Eval Premium software where measured strains are interpolated with 20 linear steps. Since the IHD technique is considered a semi-destructive technique whereby execution of the tests is known to affect the reliability of the measurements [4], at least two repeatability trials have been conducted.

Contour method (CM): The CM stress analysis was performed across the sample centre in the $\mathrm{x}$-direction as per the schematic in Fig. 1. The wire electric discharge machine (EDM) cutting used a $0.1 \mathrm{~mm}$ diameter uncoated brass wire, and a sacrificial material was bonded to the top surface to improve the near surface data quality. The cut surface profile was measured using a laser probe with a resolution of $25 \times 25 \times 0.15 \mu \mathrm{m}^{3}$. Data smoothing was implemented using an enhanced polynomial fitting routine which used a $3 \mathrm{~mm}$ smoothing mask. The residual stresses were computed by finite element analysis (FEA) which used a mesh resolution of $0.2 \mathrm{~mm}$.

\section{Results and Discussion}

The repeatability between samples was first evaluated by XRD with line scans in the x-direction as indicated in Fig. 1 with $0.5 \mathrm{~mm}$ step increments and a $1 \mathrm{~mm}$ aperture. These results are provided in Fig. 2a which shows the stresses in the y-direction. The results in Fig. 2 refer to various samples prepared in an identical manner indicated by a letter in the legend of each graph. 
The stresses vary in a range between -480 and $-580 \mathrm{MPa}$ over the $10 \mathrm{~mm}$ LSP region, where the average for each sample are more consistent with a variation from -514 to $-543 \mathrm{MPa}$. The waviness or oscillatory nature of each XRD surface stress profile is expected to be due to residual stress variations between each LSP spot impact. Each LSP spot diameter is $2.0 \mathrm{~mm}$ with a $1.57 \mathrm{~mm}$ step distance between each spot. Subsequently, a XRD line scan has been performed with smaller apertures of $0.5 \mathrm{~mm}$ and $0.2 \mathrm{~mm}$, and more distinctive variations are observed with a period corresponding to the spot overlap. Since the mean effects are consistent, the samples processing is considered repeatable. The variations of residual stress are therefore not spurious measurement phenomena, but rather physical feature due to the LSP spot overlap.

(a) Lab XRD across surface

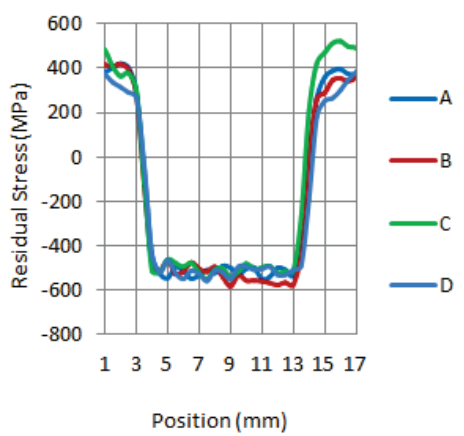

(b) Synchrotron XRD depth profiles

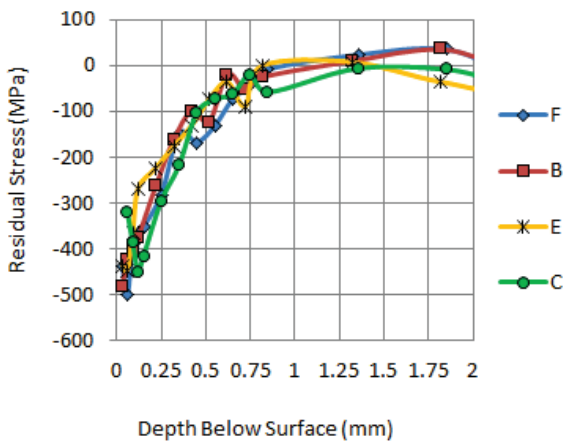

(c) Incremental Hole drilling depth profiles

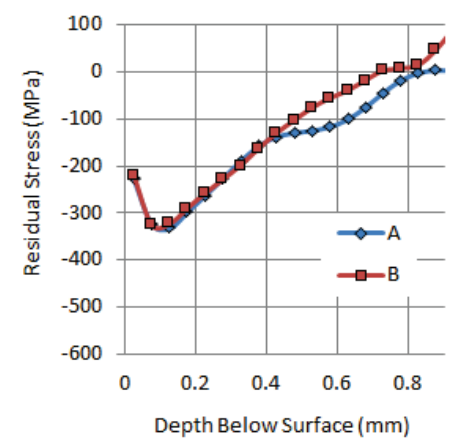

Fig. 2: Residual stress results of the LSP sample from: (a) XRD profiles across the LSP surface, (b) SXRD depth profiles below the LSP region and (c) incremental hole drilling depth profiles in the $y$-direction.

Comparisons of SXRD results from identical samples are shown in Fig. 2b. Notwithstanding the different SXRD measurements revealing similar trends, there are subtle variations between each measured profile. Previous investigations [2] on the same material processed by shot peening and evaluated on the same SXRD beamline reveal a smoother stress profile. This may suggest that the variations are not due to grain size effects, but rather potentially reflect variations within the LSP processed region. The variations between repeat samples suggest that the stress field is not as uniform as may be expected [5], which is supported by observation of the oscillation found with surface XRD measurements. If the LSP induced stress field varies according to the spot overlap, then the "needle like" gauge volume of the SXRD measurements $\left(0.05 \times 0.1 \times 1.9 \mathrm{~mm}^{3}\right)$ may be sampling slightly different areas under the LSP pattern due to minor offsets during LSP processing or measurement.

The results from the two samples for IHD are shown in Fig. 2c. There appears to be good agreement between to two tests up to around $0.4 \mathrm{~mm}$, where-after variation of the two profiles is observed beyond $0.4 \mathrm{~mm}$. Since no abnormalities were experienced during IHD execution, the repeatability within the first $0.4 \mathrm{~mm}$ suggests that the tests were executed consistently; therefore stress field variations may actually exist within the sample. The IHD results of test B shown in Fig. 2 are subsequently used to compare to other methods as the SXRD measurements were performed on the same sample. In addition, the hole eccentricity for sample B was lower at 0.02 mm compared to test A of $0.06 \mathrm{~mm}$ which implies the results of test A fall outside of the ASTM E837-13 specifications.

The CM results are depicted in Fig. 3. In order to make comparisons to the other techniques as shown in Fig. 4, a line profile down the centre is extracted, and the stresses are averaged over a 2 $\mathrm{mm}$ area (as indicated by the arrows in the top image of Fig. 3). The stress averaging provides similar dimensions to the SXRD and IHD measurements. The graph in Fig. 3 provides the 
extracted stress profiles over the $2 \mathrm{~mm}$ centre region which appears as a series of continuous lines. The averaged stress over the $\pm 1 \mathrm{~mm}$ is indicated by the black round data points. The variation of the stress field is around $\pm 12 \mathrm{MPa}$ in the first $0.4 \mathrm{~mm}$ from the surface, with increasing variations with depth to around $\pm 25 \mathrm{MPa}$ from 0.9 to $1.5 \mathrm{~mm}$. Although there are uncertainties associated with each measurement technique, the variation observed by $\mathrm{CM}$ within a single sample supports the notion of stress field non-uniformities suggested upon evaluation of the SXRD results. The averaged profile indicates a depth of compressive stress in the range of 1.1 to $1.2 \mathrm{~mm}$.

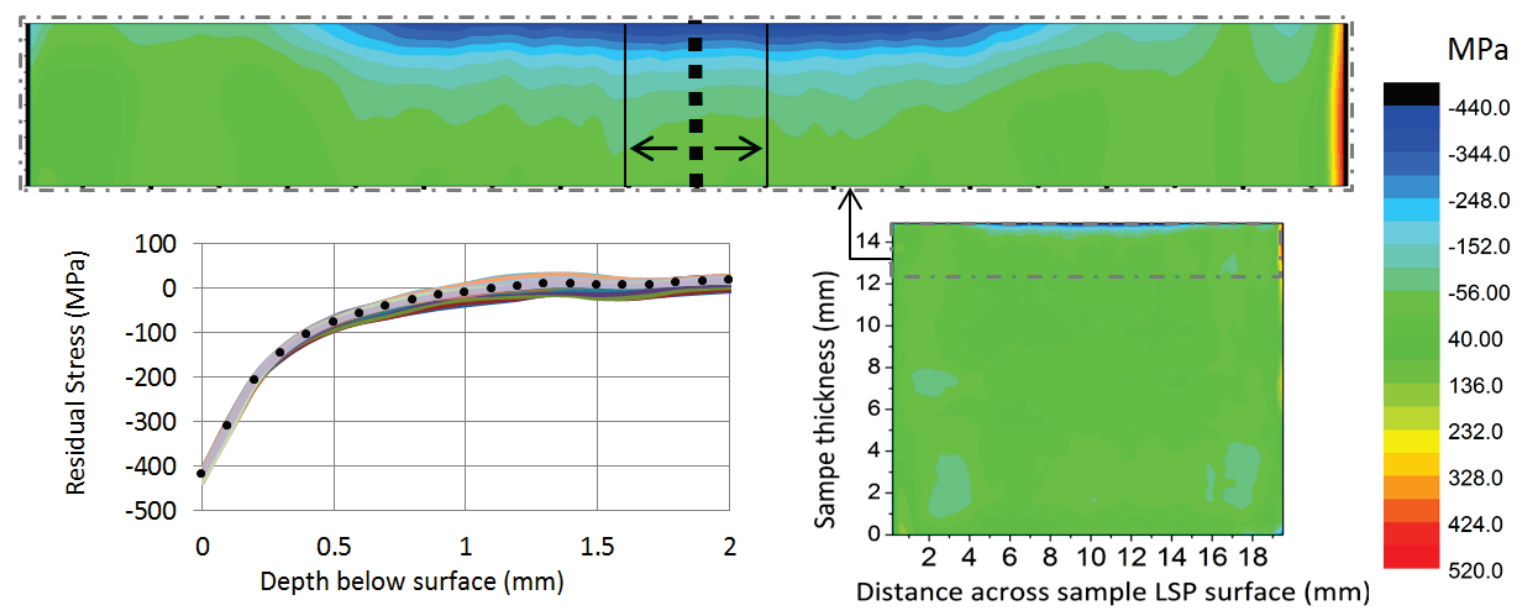

Fig. 3: Residual stress cross-sectional map of the LSP sample using the contour method on the full sample (right), a view of the first $2 \mathrm{~mm}$ (top), and a stress profile extracted from the centre of the sample $\pm 1 \mathrm{~mm}$ (mean over $\pm 1 \mathrm{~mm}$ stress indicated by round black points).

Comparing the XRD and SXRD measurements as depicted in Fig. 4, the surface stress state is similar, however the XRD depth of compressive stress obtained by electro-polishing results in a transition to tension at around $0.8 \mathrm{~mm}$ compared to beyond $1 \mathrm{~mm}$ for the SXRD results as seen in Fig 2b. Although this is plausible, especially considering the existence of a non-uniform stress field as revealed by the contour measurement, it is possible that the material removal correction factor needs to be evaluated, as only the central section of the coupon has been LSP processed although the entire surface is removed by electro-polishing.

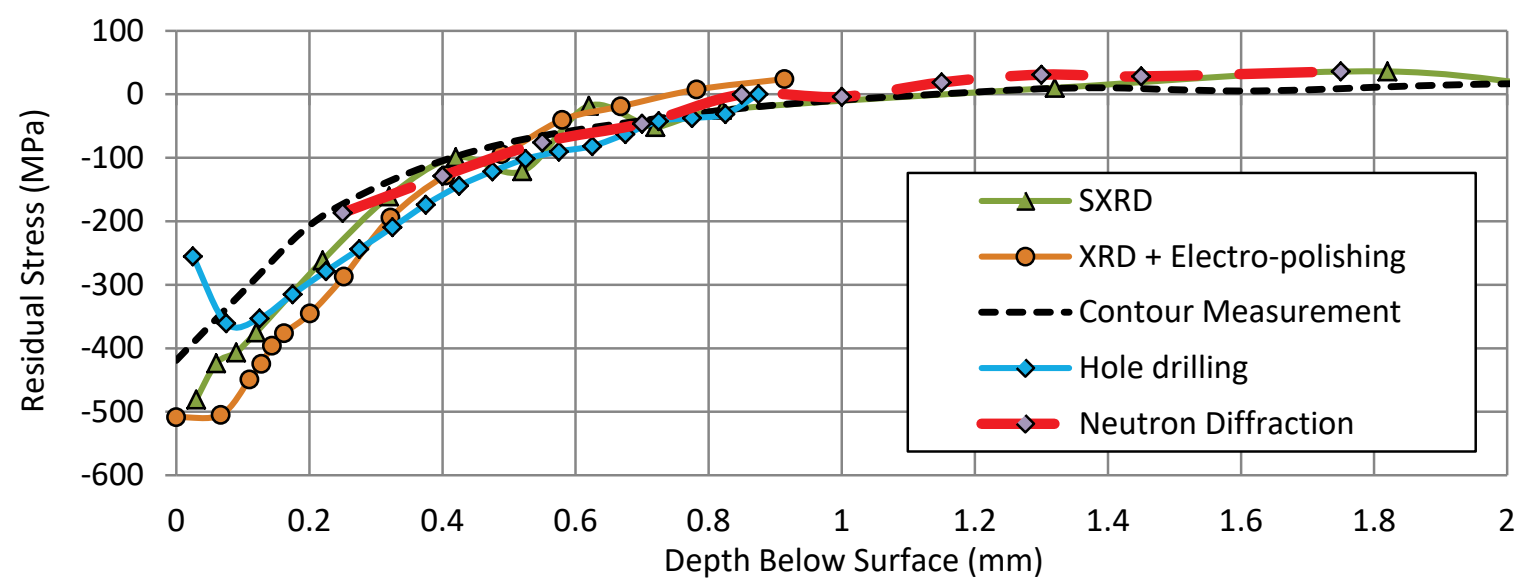

Fig. 4: A comparison residual stress profiles measured on the LSP sample using the different measurement techniques covered in this manuscript. 
The mean stress profile of the contour measurement and the ND results both agree well with the SXRD with respect to the indicated depth of compressive residual stress. The surface residual stress state indicated by XRD is $-508 \mathrm{MPa}$, however the surface stress state found by the CM method is slightly lower at $-419 \mathrm{MPa}$. The XRD results shown in Fig. 2a revealed a range of around $100 \mathrm{MPa}$ (-480 to $-580 \mathrm{MPa}$ ), therefore the surface stress result found using the contour method is not a significant variation. However, both IHD tests revealed a significantly underestimated surface stress state (of around -255 MPa) compared to the other techniques. Typically the IHD technique is not as well suited to surface stress state quantification for a number of reasons such as accuracy in surface detection, and uniform material removal in the first few steps. In addition, consideration needs to be given to the difference in sampling area or volume of the different techniques which becomes important specifically if the stress field is not perfectly uniform.

\section{Conclusions}

Various residual stress measurement techniques have been employed in order to quantify residual stresses introduced by LSP for applications of LP steam turbine blades. Each of the techniques considered provided a similar indicated depth of compressive residual stress, however variations in the near surface stress state were observed which highlights the value of utilizing a complimentary technique such as XRD which is a better suited surface stress measurement. A particular finding of this study was the presence of a potentially non-uniform stress field which is possibly due to the low overlap of the LSP spot array. Variations in the stress field were detected using fine resolution offered by SXRD and a full field map by the contour measurement. It is therefore beneficial to consider the potential stress averaging effect associated with a particular measurement technique as a selected technique may not necessarily reveal stress field nonuniformities.

\section{Acknowledgements}

The authors would like to acknowledge key contributions of Dr Thomas Buslaps at the ESRF, and Jeferson Oliveira of the Open University regarding contour measurements. In addition, the authors are grateful to Dr Axel Steuwer for GSAS data analysis regarding SXRD measurements.

\section{References}

[1] K. Ding and L. Ye, Laser shock peening performance and simulation, Woodhead Publishing Limited, Cambridge, England, 2006. https://doi.org/10.1201/9781439823620

[2] M.N. James, M. Newby, D.G. Hattingh and A. Steuwer, Shot-peening of steam turbine blades: residual stresses and their modification by fatigue cycling, Procedia Eng. 2.1 (2010) 441451. https://doi.org/10.1016/j.proeng.2010.03.048

[3] M.G. Moore and W. P. Evans, Mathematical correction for stress in removed layers in Xray diffraction residual stress analysis. No. 580035. SAE Technical Paper, 1958.

[4] P.V. Grant, J.D. Lord and P.S. Whitehead, The Measurement of Residual Stresses by the Incremental Hole Drilling Technique. NPL Materials Centre. Measurement Good Practice. Guide. 53 (2006)

[5] M. Newby, D. Glaser and C. Polese, Laser Shock Peening Process Development for Turbine Blade Refurbishment Applications Using a Commercial "Mid-Range" Energy Laser", 6th ICLPRP, Skukuza, SA. (2006)

[6] M. T. Hutchings, P. J. Withers, T. M. Holden and T. Lorentzen, Introduction to the characterization of residual stress by neutron diffraction, CRC press, 2005. 